\title{
Enhancing TCP over HSDPA by Cross-Layer Signalling
}

\author{
M. Fiorenzi*, D. Girella*, N. Möller*, A. Arvidsson ${ }^{\dagger}$, \\ R. Skog ${ }^{\dagger}$, J. Petersson ${ }^{\dagger}$, P. Karlsson ${ }^{\dagger}$, C. Fischione ${ }^{\ddagger}$, and K. H. Johansson* \\ ${ }^{*}$ KTH, School of Electrical Engineering, Stockholm, SE-100 44, Sweden \\ Email: $\{$ niels|kallej\} dee.kth.se \\ $\dagger$ Ericsson AB, Stockholm, Sweden \\ Email: \{Ake.Arvidsson|Robert.Skog|Justus.Petersson|patrik.j.karlsson \} @ericsson.com \\ ‡University of California at Berkeley, EECS Department \\ Email: fischion@eecs.berkeley.edu
}

\begin{abstract}
A comprehensive performance evaluation of a crosslayer solution to increase users' downlink data rates over HSDPA is provided. The solution consists of a proxy entity between a server and the Radio Network Controller, and cross-layer signalling from the base station to the proxy. The performance of the solution is evaluated though a detailed $n s-2$ simulator environment, which includes all HSDPA features, as well as some existing TCP enhancing protocols widely adopted for internet traffic over wireless links. Numerical results show that the proxy significantly increases the users' throughput, while also improving the utilization of the radio resources.
\end{abstract}

\section{INTRODUCTION}

The Transmission Control Protocol (TCP) is the dominating transport protocol on the wired Internet. It is starting to play an important role also in the wireless section of the network. Third generation mobile radio systems, and enhancements such as High Speed Downlink Packet Access (HSDPA), are developed to enable wireless access to the Internet. However, large fluctuations in the delay of data delivery and in the bandwidth available to users are still present, which makes efficient use of TCP over HSDPA difficult.

Designing improvements to TCP over wireless, and predicting their performance, has been a subject of intense research in recent years [1], [2]. The main approaches proposed can be basically grouped in three categories: end-to-end solutions, physical layer solutions, and cross-layer solutions. In the following, we limit the discussion to some of the relevant contributions.

End-to-end solutions suggest interactions between the sender and the receiver in order to distinguish packet losses due to the wireless channel from those due to network congestion. One of the most popular proposals is TCP Eifel [3], which adds extra information to the acknowledgement packets to recognize spurious time outs. A major disadvantage of endto-end approaches is the fact that existing TCP versions must be replaced.

This work was partially supported by European Commission through the Network of Excellence HYCON, contract number FP6-IST-511368, by the Swedish Foundation for Strategic Research, and by the Swedish Research Council. M. Fiorenzi and D. Girella were partially supported by Centre of Excellence DEWS of the University of L'Aquila, Italy. C. Fischione was with $\mathrm{KTH}$, Stockholm, when this work was done.
Link-layer approaches mainly deal with the wireless interface. The Snoop protocol [4], [5] has been proposed to deal with the negative effects of Automatic Repeat reQuest ARQ over TCP. Modelling of TCP behavior over wireless links is considered in [6], [7]. In [8], the investigation concerned the forward link power allocation and rate adaptation for TCP throughput maximization in a WCDMA system. In [9] a control framework was presented to model nested loops related to the control mechanisms of TCP, ARQ and outer-loop power control for a single user in a WCDMA scenario. The negative effects of existing TCP solutions over HSDPA systems, with focus on the physical layer, have been studied in [10].

Cross-layer approaches propose to use cross-layer signalling in the protocol stack [11]. In [12], a joint optimization of the congestion window and radio power is proposed via dual decomposition, where dual variables are used as cross layer signalling. In [13], feedback from the Radio Network Controller has been included in the design of a cross-layer proxy. Cross-layer approaches seem to be the most promising way to exploit the large number of interacting factors involved in TCP over wireless. However, cross-layer signalling may cause undesired side effects [14], and may be expensive in terms of resource utilization. Sophisticated cross-layer interactions could be hard or impossible to implement in existing communication systems.

In this paper, we restrict our attention to the HSDPA system, and consider a cross-layer proxy solution for TCP over wireless links. The proxy adapts its congestion window according to a control algorithm, which is based on feedback from the Radio Network Controller about the bandwidth over the wireless interface. The stability of the controller was established in [13] and [15]. The original contribution of this paper is a performance evaluation through an accurate simulation environment which take into account all the HSDPA characteristics. The cross-layer proxy, the Reno protocol and two of the most popular TCP over wireless solutions, namely Eifel and Snoop, are included. The performance of the cross-layer proxy are evaluated with respect to these existing solutions, and as function of the communication environment, user location, HSDPA scheduler, and cross-layer signalling.

The remainder of the paper is organized as follows: in 
we have picked up a reference one, and we have measured the throughput experienced for the downloading of a file during a session of $15 \mathrm{~s}$. The session is long enough to account for all the variations of the link bandwidth. We set a queue size of $q_{\text {ref }}=36$ IP packets. Furthermore, we specified a radio block error probability target of 0.1 .

\section{B. Simulation Results}

In Fig. 4, the throughput experienced during the downloading session at the UE is plotted as function of time and for each of the TCP implementations, and for the pedestrian environment with the reference UE at $450 \mathrm{~m}$ from the BS. The available link bandwidth, which is a ceiling to the TCP throughput, is also reported. Notice that, since the downloading session is very long with respect to the fluctuations of the link bandwidth, the throughput distribution is a sufficient statistic. It can be observed that both RNF-PROXY and RNF$\mathrm{PROXY}+$ follow quite well the variations of the link bandwidth, while other implementations have deep drops. This can be explained by looking at the congestion windows in Fig. 5: while the congestion windows of the RNF-PROXY and RNFPROXY+ exhibit a smooth behavior, those of the other implementations have large fluctuations due to the fast variations of the bandwidth. Note that the congestion windows of the RNF-PROXY and RNF-PROXY+ are taken at the proxy, while the remainder at the server. Indeed, the congestion windows of the RNF-PROXY and RNF-PROXY+ cases measured at the server are basically in congestion avoidance all the time during the file download.

The qualitative considerations done looking at previous figures can be appreciated considering Figs. 6-9, where the average throughput during the duration of the download session is reported as function of the three sampling times of the RNF. Each curve is referred to the pair TCP implementation - HSDPA scheduler. Figs. 6-7 are related the pedestrian environment, with the reference UE at $450 \mathrm{~m}$ and $800 \mathrm{~m}$ respectively. Figs. 8-9 are related the vehicular environment, with, once again, the reference UE at $450 \mathrm{~m}$ and $800 \mathrm{~m}$, respectively. In each figure, the flat thicker line at the top is the average available link bandwidth for the UE of reference, as it is measured at the BS. Obviously, it is not dependent on the sampling times of the RNF. As a first remark, the throughput degrades as the UE is positioned far away from the BS. It is interesting to observe that in any case of the environment, position and scheduler, the throughput of the RNF-PROXY+ solution has a peak at $250 \mathrm{~ms}$. This can be explained as follows. The RNF rate corresponds to the window over which the past link bandwidth is averaged, and then provided to the RNF-PROXY, which controls the congestion window for the next future. The value of the average of the recent past of the link bandwidth is likely to be present also in the immediate future. Hence, an average of the recent past results in a prediction, and $250 \mathrm{~ms}$ seems to give the best prediction results. In the case of pedestrian environment, where the reduced mobility of the UE does not cause large fluctuations of the link bandwidth, higher RNF sampling time than $250 \mathrm{~ms}$, and until $500 \mathrm{~ms}$, do not reduce significantly the throughput. By the contrary, in the vehicular

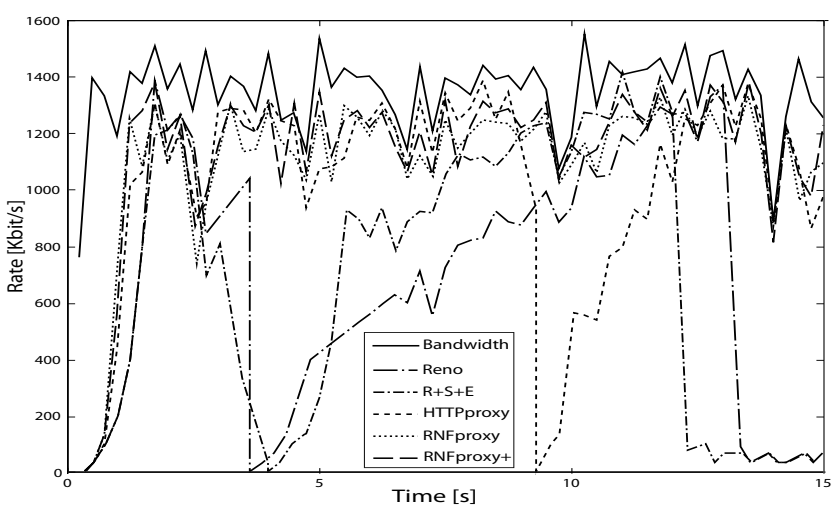

Fig. 4. Throughput for the reference UE (PedA, $450 \mathrm{~m}, 250 \mathrm{~ms}$, Max C/I).

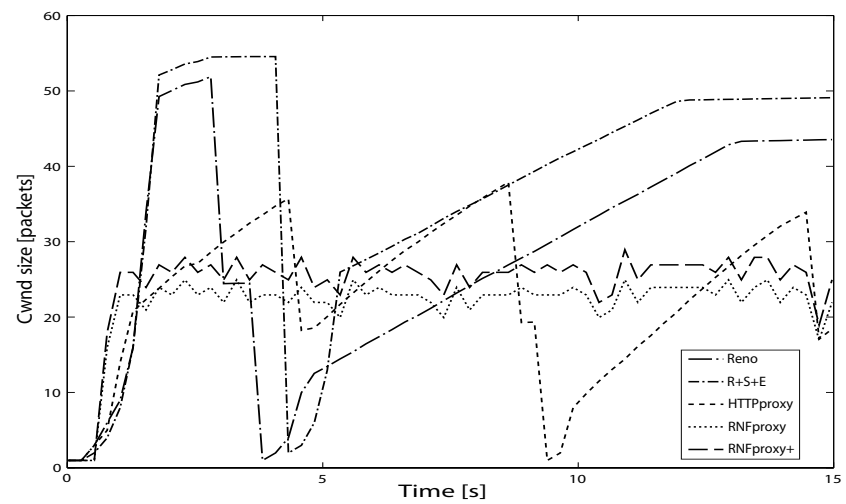

Fig. 5. Congestion window (PedA, $450 \mathrm{~m}, 250 \mathrm{~ms}$, Max C/I). For the Reno and the $\mathrm{R}+\mathrm{S}+\mathrm{P}$ solutions, the cwnd is measured at the server. For HTTPPROXY, RNF-PROXY, and RNF-PROXY+, the cwnd is measured at the proxies.

environment, where there are large fluctuations of the link bandwidth due to high mobility of the user, the throughput drops with higher slope as the RNF sampling time increases. Recall that high signalling rate of the RNF are expensive in term of resource utilization.

The RNF-PROXY, both in the simple version and with Eifel and Snoop, exhibits better performance in all the considered scenarios. Furthermore, adding Eifel and Snoop to either the RNF-PROXY or Reno, improves the throughput slightly. Looking at the effects of the HSDPA schedulers, the differences are also small. This is particularly interesting, since it implies that the BS can select the scheduler regardless of the TCP traffic, with potentially high advantages of other classes of services.

\section{CONCLUSION}

In this paper, an extensive performance characterization of a cross-layer solution for TCP over HSDPA has been presented. The solution is based on a cross-layer signalling sent from the base station to a proxy residing in the wired portion of the network. An ns-2 simulation environment for HSDPA system has been extended and implemented. The simulator includes also the Reno, Eifel and Snoop versions of TCP, as well as the cross-layer proxy. Numerical results show that the crosslayer proxy solution significantly increases the throughput experienced by the mobile user, and thus reduces the end- 
